\title{
Compartiendo los problemas de la Atención Primaria: el Foro de Médicos
}

Los médicos de Atención Primaria nos hemos reunido muchas veces, hemos protestado todavía más y, cada cierto tiempo, empezamos otra vez. Para algunos seguro que somos unos pesados, pero nosotros preferimos pensar que somos inasequibles al desaliento y que buscamos nuevas maneras de mejorar la Atención Primaria. Para ello se ha constituido este Foro de Médicos de Atención Primaria, en el que trabajamos como aliados y colaboramos con entidades muy distintas: sindicatos profesionales (CESM); la Organización Médica Colegial (OMC); dos sociedades científicas de pediatras de Atención Primaria: la Asociación Española de Pediatría en Atención Primaria (AEPAP) y la Sociedad Española de Pediatría Extrahospitalaria y Atención Primaria (SEPEAP); y tres sociedades científicas de médicos de familia: la Sociedad Española de Médicos de Atención Primaria (SEMERGEN), la Sociedad Española de Médicos Generales y de Familia (SEMG) y la Sociedad Española de Medina de Familia y Comunitaria (semFYC).

¿Y qué hemos hecho? Como objetivos generales tenemos el reconocimiento de la Atención Primaria en su papel de eje central de la asistencia sanitaria; la potenciación de las políticas de salud de consenso y comunes para todas las comunidades autónomas; el análisis de las necesidades de recursos y reorganización de su distribución en base a necesidades de salud y el abordaje de los estudios de grado y postgrado.

Trabajamos en la elaboración de áreas de mejora de la Atención Primaria, planteándonos tres tipos en función de su factibilidad y viabilidad. En primer lugar, aquellas áreas que se pueden abordar sin coste añadido y que tienen muy fácil consecución:

- Regular la burocracia de otros niveles asistenciales.

- Gestionar racionalmente la Incapacidad Temporal (IT).

- Tener libre acceso a pruebas diagnósticas (capacidad resolutiva).
- Reorganización de centros: tiempo para docencia e investigación, flexibilidad de agendas, regular y definir roles.

- Participación de los profesionales en la toma de decisiones del ámbito sanitario.

- Disminuir dispersión de centros de salud: adecuar los recursos a las necesidades de los profesionales.

- Formación de pediatras en Atención Primaria.

- Informe de salud único y ajustado a necesidades normativas.

- Evaluar la inversión en investigación en Atención Primaria.

Sobre estas áreas hemos elaborado cinco documentos, muy básicos, pero consensuados, que nos están ayudando a que se pongan en marcha algunas iniciativas. En este momento existe un compromiso por parte del Ministerio de Sanidad, Política Social e Igualdad de trabajar con el Ministerio de Trabajo e Inmigración en medidas que mejoren la gestión de la IT en la línea que se les ha propuesto, y que estas medidas se pongan en marcha lo antes posible.

En segundo lugar, las áreas de mejora con un coste moderado y/o alcanzables a corto-medio plazo, y que resultaban relevantes para todos los integrantes del Foro de Médicos de Atención Primaria. Estas son:

- Regular la población asistida.

- Medicina de familia y universidad.

- Regularización de oposiciones y concursos.

- Homogeneizar presupuestos sanitarios en las comunidades autónomas.

- Receta electrónica.

- Desarrollar área de capacitación específica en Pediatría en Atención Primaria.

- Homologación médicos extracomunitarios.

- Evaluación continua de "Atención Primaria del siglo XXI: Estrategias de mejora” (AP21).

- Campañas de educación para la salud: uso racional de los servicios sanitarios. 
Y en las áreas de coste elevado y/o difícilmente alcanzable situamos:

- Equilibrar y redistribuir el presupuesto en AP para alcanzar un $25 \%$.

El 12 de abril organizamos el Día de la Atención Primaria, al que invitamos a todas las entidades implicadas en ella, y les pedimos su colaboración y apoyo en la elaboración de un manifiesto (http:// www.semfyc.es/es/noticias/destacadas/listado/ Manifiesto_Dia_Atencion_Primaria/), en forma de decálogo, que presentamos tanto al Ministerio de Sanidad, Política Social e Igualdad como a las Comisiones de Sanidad del Senado y el Congreso de los Diputados.

Queremos que todas las comunidades autónomas repliquen este foro, algunas como Madrid ya lo han hecho, porque muchas de las propuestas e iniciativas son competencia de las comunidades autónomas. Pensamos que todo el ámbito estatal debe moverse para que los médicos de Atención Primaria nunca estemos satisfechos con la rutina, la desgana o los elementos desmotivadores, y sigamos persiguiendo siempre lo mejor para nuestros pacientes y para la Atención Primaria.

Ana Pastor Rodríguez-Moñino Vicepresidenta de la Sociedad Española de Medicina de Familia y Comunitaria

Josep Basora i Gallisà

Presidente de la Sociedad Española de Medicina de Familia y Comunitaria 